"Electric Vehicle Assistant Based in Driver Profile"

International Journal of Electric and Hybrid Vehicles, vol.6, no.4, pp.335-349, 2014.

http://www.inderscienceonline.com/doi/abs/10.1504/IJEHV.2014.067626

ISSN: $1751-4088$ (Print)

ISSN: 1751-4096 (Online)

DOI: 10.1504/IJEHV.2014.067626

This material is posted here according with:

"Posting the Author's Original* or Accepted Manuscript* on the Author's personal web pages and/or institutional repositories and/or subject repositories without embargo, or the Proof* upon condition that it shall not be accessible until after six months from Inderscience's publication date."

(C) 2014 Inderscience's 
João C. Ferreira, Vítor Monteiro, João L. Afonso, "Electric Vehicle Assistant Based in Driver Profile" International Journal of Electric and Hybrid Vehicles, vol.6, no.4, pp.335-349, 2014. ISSN: 1751-4088 (Print) 1751-4096 (Online), DOI: 10.1504/IJEHV.2014.067626

\title{
Electric Vehicle Assistant Based in Driver Profile
}

\begin{abstract}
This paper presents the outcomes of a research work consisting in the development of an Electric Vehicle Assistant (EVA), which creates and stores a driver profile where are contained the driving behaviours related with the EV energy consumption, the EV battery charging information, and the performed routes. This is an application for mobile devices that is able to passively track the driver behaviour and to access several information related with the EV in real time. It is also proposed a range prediction approach based on probability to take into account unpredictable effects of personal driving style, traffic or weather.
\end{abstract}

Keywords: Electric Vehicle, Range Prediction, Data Mining, Range Anxiety, Driver Profile, Mobile Device Sensor Data

\section{Introduction}

Nowadays, mobile devices play a ubiquitous role in accessing and processing information in real time, with good bandwidth at low prices and with a diversity of integrated sensors that makes them capable of generating information with high accuracy and precision. This sensing capacity is combined with easy programmability and interaction, and due to the good market penetration makes these devices good for unobtrusive monitoring of user motion habits. So our intention is to apply these features to capture the driver behaviour in an EV and create a driver profile where is stored their past behaviour. In this profile is stored the information related with the route performed considering the schedule, trajectory, EV speed, EV energy consumption, and also information related with the EV battery charging process. These variables are used by the proposed system to help to give personalized information to the EV driver related with the EV usage. This is done aiming planning the EV trip and their usage optimization, including the EV energy use and the battery charging process. Therefore, through the developed application, the EV driver can interact with the EV battery charging system and combine this information with about public charging stations. This information is related with the charging station position, the availability, the energy price, and the reservation with the associated 
parking place. The integration of this information associated with driver interaction devices and applications, it will contribute for a better trip planning and EV energy usage. Consequently, it is reduced the range anxiety of the EV driver, which is related with the driver fear to stay without energy in the EV battery and in a place where is no possible perform the battery charging process. In this research work we describe also a personalized range prediction in a mobile application.

In a first stage is obtained an estimation of the EV range, and after that is started the calculation of the route optimization based on the EV current position. In order to optimize the route, this process is interactive. This approach can be complemented with a personalized one using a driving profile that acts as a training set for a Data Mining (DM) approach to estimate the EV range autonomy. The DM approach uses a regression model to find the best fitting estimation. This is done based on the current battery State-of-Charge (SoC) level, and the past driver behaviour (where is included the battery SoC level, weather information related with wind and temperature, EV average speed, and traffic information). The output of this approach is an individual range prediction based on past driving data combined with external factors like traffic information and weather (sky conditions and temperature). This output is obtained through a Naïve Bayes approach, where it is fitted the past driver behaviour to current situation in order to estimate a more accurate EV range based on the driver behaviour. This range prediction, which is represented on a map, is a useful information for the EV driver. Therefore, it is possible check if the desirable destination could be reached with or without extraordinary driving optimization actions (e.g., range could be increased turning off or reducing the air-conditioner, or smooth the driving style). Also this approach can be used to estimate the EV battery State-of-Health $(\mathrm{SoH})$ in terms of battery SoC based on past charging experience and lifetime in a similar process. In the user interface, checkboxes and user-defined entries could be added in order to manually specify trip features (e.g., air-conditioning will be used, and what is the desired cruising speed).

The mobile application, which allows obtain a personalized range prediction and its representation, as well as the integration and interaction with public charging stations, is called Electric Vehicle Assistant (EVA). Thus, EVA is a mobile application used to help to increase the driving autonomy through the reduction of energy consumption and driving efficiency. Through the integration and interaction with the public charging stations, the EV drivers can plan his journey considering the charging stations position, and booking a charging point for a specific time period. Therefore, the distance that the driver can drive comfortably without fearing 
running-out the battery is increased. The main application modules of the EVA, illustrated on Figure 1, are:

1. EV battery charging functions, divided in Home Charging (HC) and Public Charging Stations (PCS), described in section 2.

2. Vehicle Interface System (VIS), where we develop a basic electronic unit that can accommodate the different interfaces required for this system (CAN-bus, Bluethooth, 3G, Wifi), described in section 3.

3. Driver Profile, where we create and store in a SQL Server database the driver's profile, check the main EV energy consumption parameters and all the data collected from the mobile device sensors, e.g., GPS and aceelerometer, described in section 4 (Driver Profile).

4. Range Prediction Process, which is detailed described in section 5.

5. Extended Range Navigation (ERN), which is an application to give to the EV driver information about a combined navigation strategy between consumption optimization and travel speed, described in section 6 .

6. External System Interface to public transportation, energy market and points of interests (POI), described in section 7.

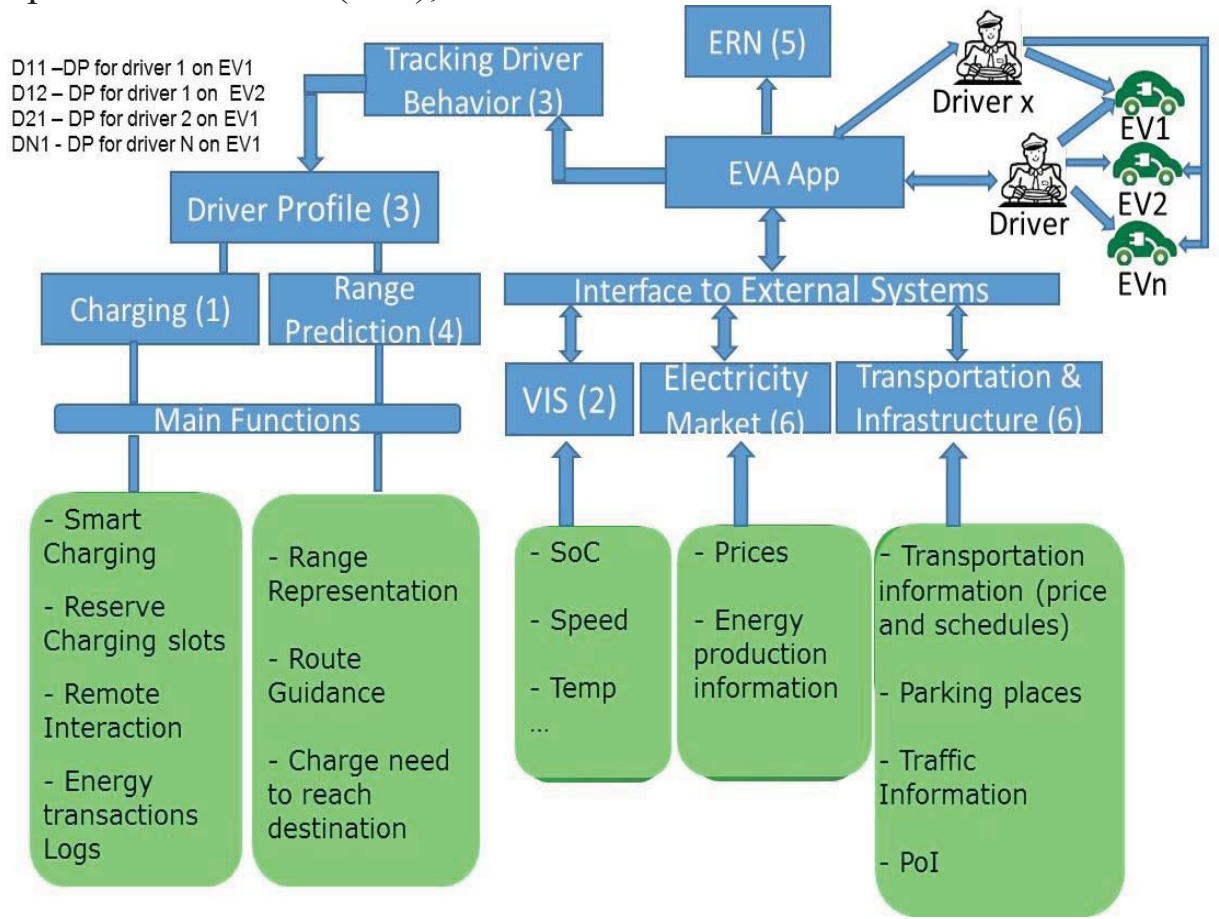

Figure 1: Electric Vehicle Assistant (EVA) main application modules. 


\section{Charging Functions}

Taking into account the EV battery charging process, this application is mainly focused in the Home Charging (HC) and Public Charging Station (PCS).

The Home Charging (HC) consists in charging the EV battery at home, where the $\mathrm{EV}$ is plugged in a socket to perform the battery charging process without any concern about the contracted power for the home. This battery charging process can be external controlled, allowing the interaction through remote control communication. This is important in order to establish a smart battery charging strategy, choosing the convenient periods (where the energy is cheaper) and, where is considered the home current limitations and the power grid limitations [1]. This issue is already identified in literature in [2][3]. Moreover, this battery charging process can also be controlled by a Collaborative Broker, which also deals with local micro generation [4].

On the other hand, the main goal of the developed application for Public Charging Station (PCS) is the driver guidance towards the nearest PCS, and the enable of online advanced booking of charging spots. Once the application can estimate the charge prediction (sections 5 and 6) based in the battery SoC level, the driver profile, and the remaining distance, the application can find the minimum value of energy to charge the batteries, and consequently reserve the PCS for a specific schedule. This strategy is extremely important to the EV driver aiming reduce its range anxiety and to make possible reach the desired destination in the less time possible.

\section{Vehicle Interface System}

Taking into account all the necessary data transfer between the vehicle and the external system, the complexity of the inner-vehicle architecture must be abstracted from the high-level applications. In order to accomplish this goal, we are working in a definition of a common API for "Vehicle2Infrastruture" (V2I). At this moment we have an On-Board Unit (OBU), that provides locally (in the EV) and remotely (to mobile devices applications) relevant data communications. The OBU device is based on a microcontroller that integrates CAN-bus communication, Bluetooth, GSM/GPRS (Global System for Mobile communications / General Packet Radio Service) and GPS (Global Positioning System). Therefore, the implementation of the CAN-bus protocol allows requesting and receiving data from the EV. With the available OBU wireless communication interface, it is possible to report both locally and remotely the data through Bluetooth and/or GSM/GPRS technologies, respectively. Moreover, Bluetooth allows the OBU integration with mobile equipment, such as 
mobile/smart phones. The OBU interacts through the Battery Management System (BMS), which is an equipment that has several characteristics, for instance, equalize the charge across all the batteries and estimate the battery SoC. In our case this estimation is based in the voltage batteries, and is useful to determine the EV range prediction.

The aforementioned Vehicle Interface System (VIS) allows measuring the key performance indicators from each event driven by the vehicle through the CAN-bus on-board data collection system. Then, those variables are crossed with publicly available historical meteorological data, in order to build a driver profile and to find the most important parameters related with driver efficiency.

\section{Driver Profile}

The driver can be educated to understand how the EV works regarding energy consumption. It is important to adapt the message given to the driver, so that he or she can easily assimilate the different strategies for the optimization of energy saving. The main idea is to store their behaviour in a driver profile that can be used for range prediction, for alerts towards energy savings or even for charging strategies taking into account the distance usually performed. The driver profile plays an important role in the range prediction process, and is based in three main components:

1. Drivers trip information, such as data/time (work days, weekend, holidays, start time, and finish time), distance (in $\mathrm{km}$ ), and battery SoC level;

2. EV characteristics, like model, year, battery charger, battery type and capacity;

3. EV driving style.

As illustrated in Figure 1, each driver has associated a profile in every vehicle or $\mathrm{EV}$ that he drives.

\subsection{Sensors Data from Mobile Device}

The interaction of the sensors with the mobile device is dependent of the operating system. As aforementioned, in this project was used Android due to the available framework. This framework provides several classes and interfaces that are useful to perform applications. The developed application detects the available sensors and in an application menu shows to the user the available sensors and the option to select each one as well as the sampling time interval. Taking into account that our application was developed for Android 4.0, we have the following sensors available: Accelerometer; Temperature; Gravity; Gyroscope; Linear Acceleration; Magnetic field; Orientation; Pressure; Humidity; and Rotation. 
This sensor information is locally stored in a little SQL data base and later transmitted to a cloud storage. This data provides useful information for mobility process. In this process is important the user put the mobile device in the correct position in the car, to keep the reference system, as shown in Figure 2. In a new version we will try, in the begging of acquisition process, detect the mobile device position and use this position as reference. For now we are using a pre-defined position and the user should respect this for the correct data acquisition.

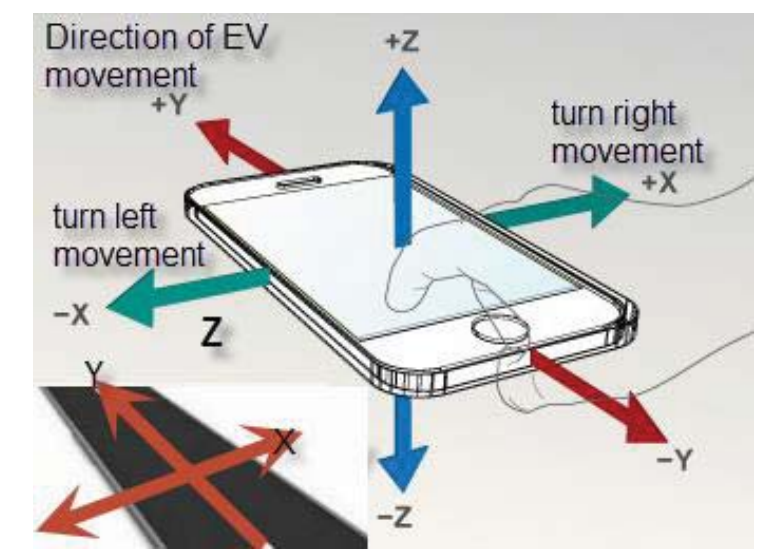

Figure 2: Mobile Device orientation for correct data acquisition.

\subsection{EV Driving Style}

Any time a driver starts and stops a voyage, the application detects this situation based on accelerometer data and starts the acquisition process. We acquire the GPS position and the acceleration in the three axis. From this data it is possible is possible to define acceleration, breaking and inertial travelling events, as presented in Figure 3 (a) (Y-axis). Breaking and acceleration can be divided in normal and excessive. An excessive acceleration or breaking event is a configurable parameter, which we define as a change on $\mathrm{Y}$-axis acceleration change more than $2 \mathrm{~m} / \mathrm{s}^{2}$ from previous measurement. Also information from (X-axis), as presented in Figure 3 (b), is used to define drive style based on the rightlleft turn performed. Aggressive turns (right and left) with acceleration and breaking can be helpful to classify the driving style as aggressive. External data obtained from CAN-bus through VIS interface are: (1) Air conditioner usage; (2) Lights usage; and (3) Battery SoC level. All of this data is related with the $\mathrm{EV}$ driving consumption parameters. It is possible to characterize the driver behaviour in terms of this driving parameters. 

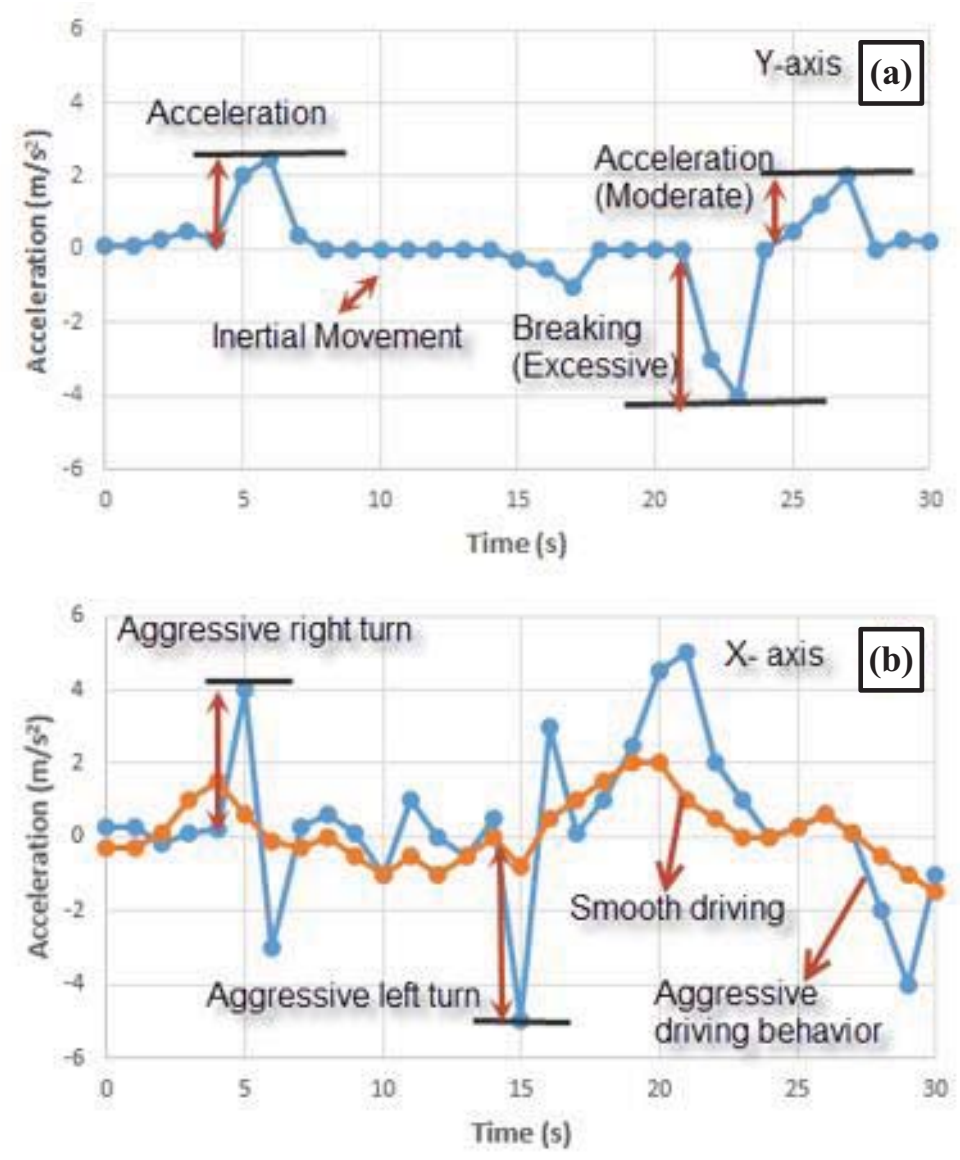

Figure 3: Accelerometer data acquisition for: (a) Y-axis; (b) X-axis.

\subsection{EV Energy Consumption}

The aim of this task is to look at the different EV systems under the energy consumption perspective, in order to provide information for a realistic range prediction of the travel. Models for the energy consumption of the vehicle, as well as regenerative braking potential, will be developed for the different operating conditions. Two types of energy should be characterized: electrical and mechanical. In terms of electrical energy consumption, the various powertrain components (batteries, BMS, inverter, controller, and motor) and auxiliary systems (air conditioner, lights and other electric-driven devices) were analyzed from CAN-bus data. In terms of mechanical energy consumption, the analysis was performed from the data available of driving style, and were studied the most influential parameters for the energy losses during driving. This study was performed based on real data taken from an EV developed at ISEL, in the Lisbon area: the electric vehicle VECCO [www.veeco.pt]. Additionally, historical 
meteorological data was obtained from the site Weather Underground [wunderground.com], assuming a unique point as representative of the regions of Lisbon. Collected variables examples are: temperature, visibility, wind speed, rainfall, and weather events (as storm and heavy rain). All the data collect from the VIS interface, mobile device sensor data and weather information were stored in a Microsoft SQL Server Analysis. It was used a CRoss Industry Standard Process for Data Mining (CRISP-DM) methodology [5], illustrated in Figure 4:

- Process 1. Data collection process performed in 2013 of driving characteristics that are storage in a SQL database. Table I identifies the 20 parameters collected and used for our study from mobile device sensor data, VIS interface.

- Process 2. Pre-processing data process involves the identification of outliers ${ }^{1}$ to reduce the number of records. Inconsistent data was also removed from outliers' identification approach in an outlier's implementation approach [6];

- Process 3. Online Analytical Processing (OLAP) process [7], was used to give an overview of data available and representation as shown in Figure 6;

- Process 4. Data Mining process was used to identify the Key Performance Indicators (KPI) towards energy savings and to perform the range and charge prediction process. This process is described in section 7.

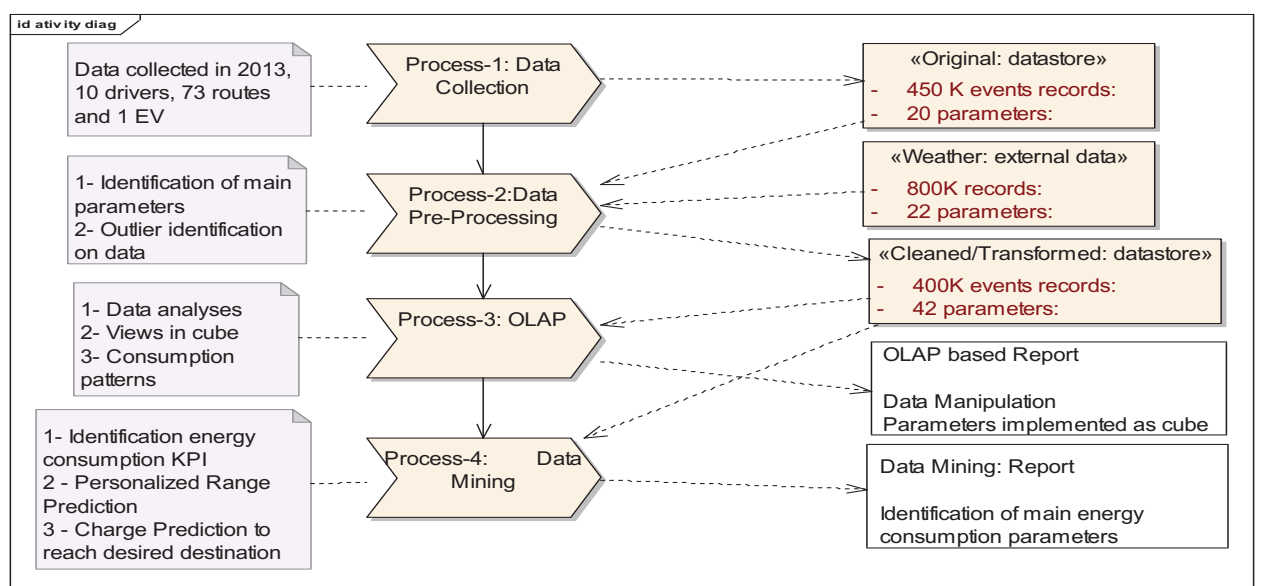

Figure 4: Key influencing factors for average energy consumption.

From this approach was possible to identify key energy consumption actions for each driver. From this type of analysis, the system identifies

${ }^{1} \mathrm{~A}$ outlier is an observation point that is distant from other observations due to measurements errors. 
actions to reduce energy consumption, and automatically alerts the driver. However, no automatic actions are performed, because the ultimate decision of reducing or not energy expenses is made only by the driver. Thus, the driver may accept or not the advising to turn off the air-conditioning or to reduce his driving speed. All the data regarding alerts and driver's response is stored in a driver profile. From this analysis (Data Mining in process 4), the highlighted top four key influences are: the utilization of the air conditioning; the number of excessive braking events; the maximization of the inertial movement; and also the number of excessive acceleration events.

Energy saving alerts are produced through the EVA application taking into account the energy consumption study performed. For example, if the desirable destination is in the limit of the predicted range, the system can detect that the air conditioning is on, and suggests to the driver to turn it off, or he will have to perform a battery charging operation during the trip.

Table I: Operational (and collected) data from CAN bus events and mobile device sensors

\begin{tabular}{|c|c|c|c|c|c|}
\hline $\begin{array}{c}\text { CAN-bus } \\
\text { Collected } \\
\text { Variables }\end{array}$ & \multicolumn{2}{|c|}{ Mobile device sensor data } \\
$\begin{array}{c}\text { Usage event } \\
\text { per } 100 \mathrm{~km}\end{array}$ & $\begin{array}{c}\text { Excessive } \\
\text { events per } \\
100 \mathrm{~km}\end{array}$ & Date & Identification \\
$\begin{array}{c}\text { Battery } \\
\text { SoC Level }\end{array}$ & $\begin{array}{c}\text { Inertial time } \\
\text { travel (\%) }\end{array}$ & Acceleration & Acceleration & Day & Voyage number \\
$\begin{array}{c}\text { Air } \\
\text { Conditioning } \\
\text { Usage }\end{array}$ & Speed & Brake & Brake & $\begin{array}{c}\text { Time } \\
\text { (h:m:s) }\end{array}$ & $\begin{array}{c}\text { EV Id (e.g. } \\
\text { weight and } \\
\text { battery capacity) }\end{array}$ \\
\hline $\begin{array}{c}\text { Light Usage } \\
\text { Windshield } \\
\text { Usage }\end{array}$ & Location & Traffic & & Month & Route Variant \\
\hline
\end{tabular}

\section{Range Prediction Process}

Range prediction has already taken the attention of scientific community, where can be found several published works [8][9]. Also Data Mining (DM) approaches have been applied for the estimation of energy consumption [10][11]. Driving range is intensively related with the driving style or mode. This happens in all types of vehicles, but on EVs, due to the weakness related with the amount of energy stored on-board, this relation is much clearer. Thus, changing driving style and driving habits may be a 
considerable factor on energy saving and on extending the EV autonomy. Considering that the actions or driving habits can bring significant energy savings to the vehicle operation, it is important to evaluate how receptive EV drivers will be towards the achievement of the intended energy saving. This type of actions may be accepted for some of the driving process, but not for all driving processes. For example, one driver may accept the vehicle control system to automatically turn off the air-conditioning under certain conditions, but will not accept the system to limit his driving speed. Part of this study will create and store a Driver Profile for further analysis. These Driver Profiles will play an important role on the project, since range prediction will be based on the assessment of the drivers' usual behaviour. The range prediction is based on three main dependency types [12]:

- The EV with its main variables: The model of the vehicle (mainly their performance under different scenarios, considering the speed and the acceleration); The chemical technology of the batteries (as lithium-ironphosphate, lithium-titanate, or nickel-metal-hydride) and its capacity; The batteries characteristics (mainly variation of the battery SoC level, lifespan, performance, specific power, specific energy, and safety); The $\mathrm{EV}$ powertrain (electric motor and power converter, as well as the other electric parts, as battery charger, controllers, and power cables). All of these EV variables will influence the battery SoC, and consequently the range prediction. The battery $\mathrm{SoC}$ and other relevant parameters are provided to the main control system through CAN-bus communication. The provided information is stored in a data base, in order to predict the available range autonomy.

- The driver behaviour: The speed, acceleration and breaking (taken from mobile device sensor information); The driver past behaviour (e.g., battery SoC level versus achieved distance, which is stored in a data base); The weight loaded in the EV (that is a manual input); The driving direction (that is acquired based on the GPS information).

- Environment: Current location of the EV; Traffic conditions; Road information (obtained through a distance graph); Weather information (wind and temperature that are taken from a web service or from an EV sensor); Altitude of the current location of the EV (taken from the GPS device); and traffic information is performed by the combination of VIS data (detection of engine on and handbrake off) to detect situation off EV stopped and then we use the current EV speed divided by the maximum road speed (available from navigation systems or google maps), this we call Traffic Relation (TR). Taking into account the information of several vehicles in the road (road is defined by two adjacent nodes in a graph), 
we use the average TR of movement vehicle in the road: If this division is above $50 \%$ we consider no traffic, but if the result vary from $15 \%$ to $50 \%$ we consider a yellow traffic situation, from $15 \%$ to $1 \%$ a red situation and below $1 \%$ a very severe traffic situation. This four classes are used in the range prediction process, described following. Figure 5 shows the range prediction process. This traffic detection is complemented with external information of traffic collected from web services.
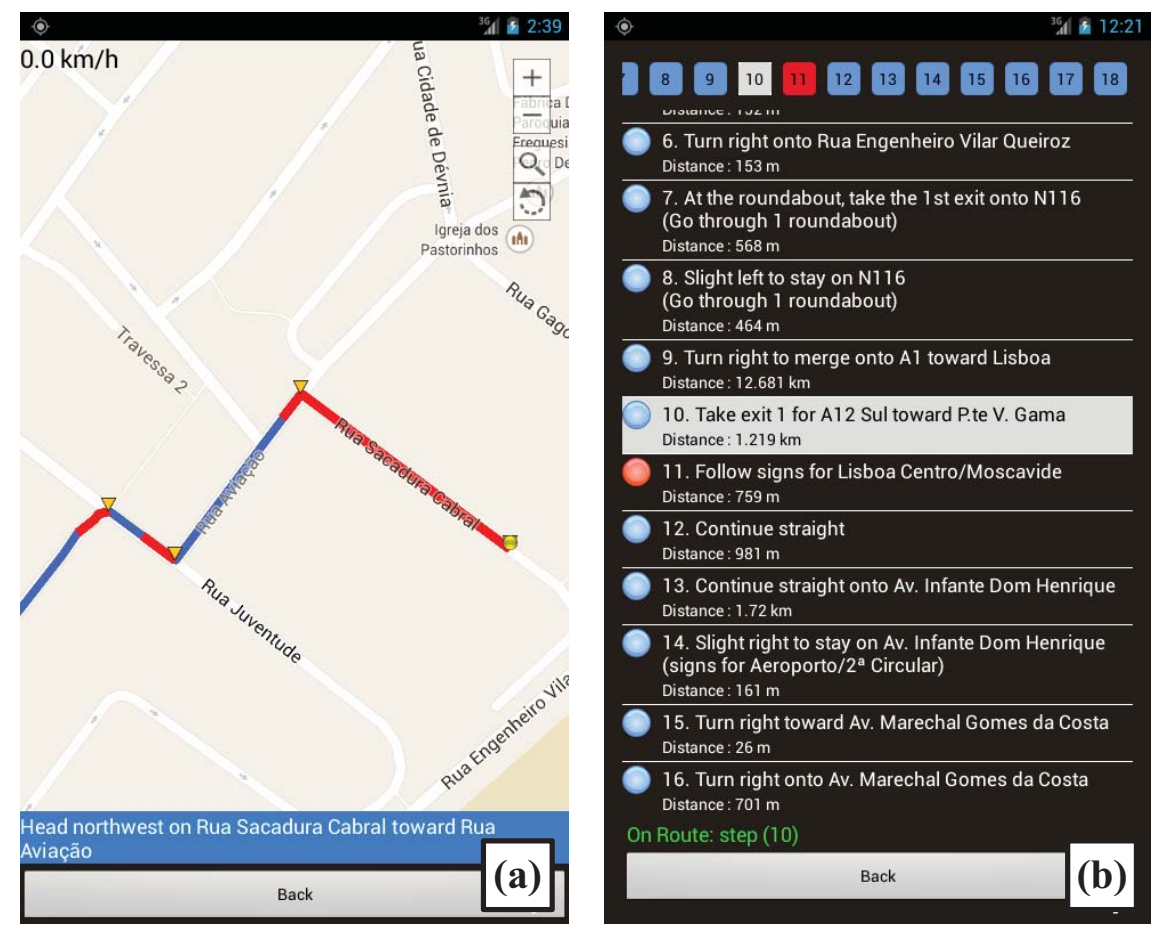

Figure 5: Range prediction process: (a) Representation of the traffic identification, where red colour means a severe traffic situation on the road node, and blue colour represents an easy traffic situation; (b) Description of the traffic information in textual mode and related route path towards final destination.

From the GPS coordinates it is easy to calculate travel distances, which are combined with battery SoC level. Driver profile is based on this data that acts as a training set for a Data Mining (DM) approach to estimate the EV range autonomy. This is a probabilistic approach since we use Naïve Bayes (NB) algorithm, using past data available that has a training set to tune probabilistic parameters. Since NB works better with discrete variables and most of our variables are continuous we allocate data into class based in a clustering approach of existing data [13]. Figure 6, shows the results of clustering process for acceleration data (from zero to 3500 events per 100 
$\mathrm{km}$ ) and breaking events (from zero to 2300 events per $100 \mathrm{~km}$ ), with the creation of seven classes. We define 7 classes, where the first include the $10 \%$ lower events (zero to 350 for acceleration and zero to 230 for breaking), the other five where defined based on the clustering method of equal areas performed by the DISCRETIZED SSAS ${ }^{2}$ process in the SQL and the last contains the upper 10\% (from 3510 to 3500 for acceleration and from 2070 to 2300 for breaking). More details are presented in [14].

In this probabilistic approach it is possible to combine other external factors, like traffic information and weather conditions of wind and temperature in order to estimate a more accurate EV range autonomy. NB uses old data to identify the effect of these parameters in the EV consumption. This range prediction, represented on a map, can be a useful information for the driver in order to check if the desirable destination can be reached with or without driving optimization. For instance, the range can be increased by reducing or turning-off the air-conditioning, or by smoothing the driving profile. This approach can also be used to estimate the EV battery lifetime, based on the past battery charging experience, in a similar process. Range and charge prediction processes use the same NB approach where the variable battery SoC and distance $(\mathrm{km})$ change the role.

\section{Extended Range Navigation}

This module takes into account the necessity of suggesting consumption efficient routes, and/or routes that considers the charging needs for the specific EV. At computing the shortest and the fastest route, the best economic routing strategy is to use a mixture of both. For EVs it may be important to optimize the route by energy consumption. This Extended Range Navigation (ERN) specification addresses mainly three different tasks:

1. Range Prediction - This application estimates the range of EV based on the current battery SoC level and its historical data. A green prediction range is calculated based on worst scenario data available in driver profiles. A yellow range prediction is calculated based on average data available on driver profile. A red range is calculated based on all possible optimization performed to save energy.

2. Charging Prediction - For a given desirable destination this application, based on historical data, calculates the necessary energy in the battery in order to the EV reach the desired destination.

3. Extended Navigation Representation (ENR) - This process is performed in three main approaches: (1) From current position and with the range

\footnotetext{
${ }^{2}$ http://msdn.microsoft.com/en-us/library/ms174512.aspx
} 
prediction calculation, a circular range is represented; (2) Using current position and based on Google Maps API, a polygon range is represented based on Erro! A origem da referência não foi encontrada.; (3) The same as in the second approach, where we take into account current traffic, weather condition and road topology.
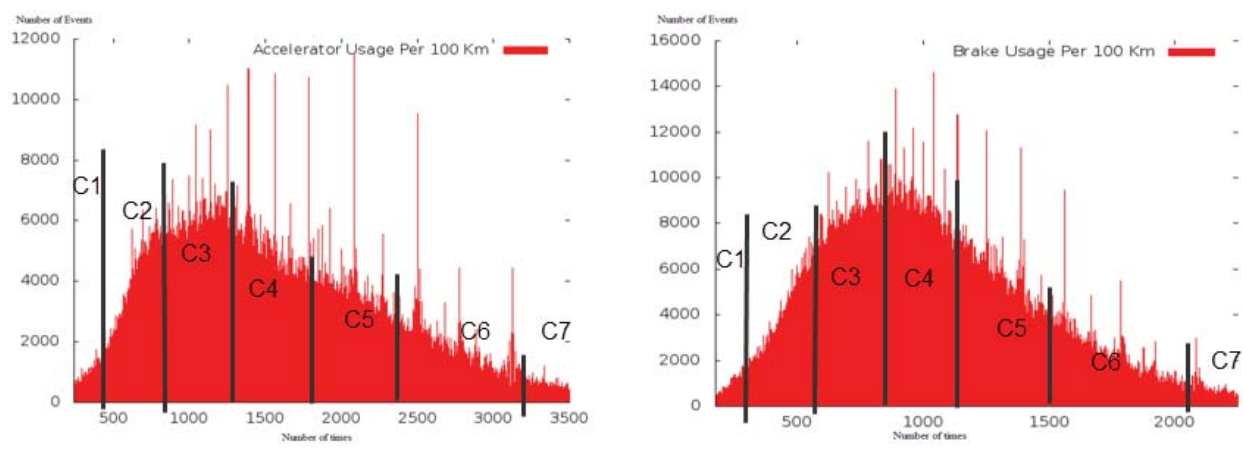

Figure 6: Distribution of data related with the accelerator usage and break usage per $100 \mathrm{~km}$, and related discretization process, classes creation and identification.

Since a mobile device has a limited process capacity, most of these complex calculations are performed remotely, in order to get the user of an EV as fast as possible, and in the most reliable way, to his/her desired destination, based on the Cloud services of:

1. Geographic Information Server (GIS), which is responsible for the data representation of the 3D topography, the vertex/node abstraction. Our GIS approach are performed based on Google Maps API.

2. Environment Server (ENV), which is responsible for the weather data, like current temperature, wind and traffic information picked from a web service, but also that is responsible for the computing and delivering of the battery charge cycles and the driving style. Residing on the Integration Platform, it uses all the data and computes all the results.

As result, the extended Range Navigation develops an approach on how to best match the data coming from the EV and the Cloud services residing on the Integration Platform, how to compute them, and finally display the best route and optimized range to the EV user.

Figure 7 illustrates the range prediction process developed for the mobile application presented in this work, where the first screen (a) shows the starting of a range prediction, in which the application presents the location of the EV. The option of specifying layers is shown in the screen Figure 7 (b). In this case, the user chooses the range prediction process, and the results are illustrated in the screen (c). A safe range is shown in green (probability of reaching above $90 \%$ ), in yellow a range which the driver is 
able to reach with usual behaviour representing a probability from $90 \%$ to $40 \%$, and in red a range that is not guaranteed to be achieved unless the drive performs driving optimization (probability bellow 40\%).

This range information can be complemented with the location of POI and Charging Stations (CS), illustrated in Figure 7 (a). In Figure 7 (b) is shown an example of alert of insufficient charge for the EV to reach a desired destination. In this case the proposed route is showed, the information about insufficient battery SoC is highlighted, and recommendations about actions to reduce energy consumption can be suggested. The application can also show options of the nearest charging station in the proposed route. Figure 8 (c) shows information about charging levels through the time and charge performed.

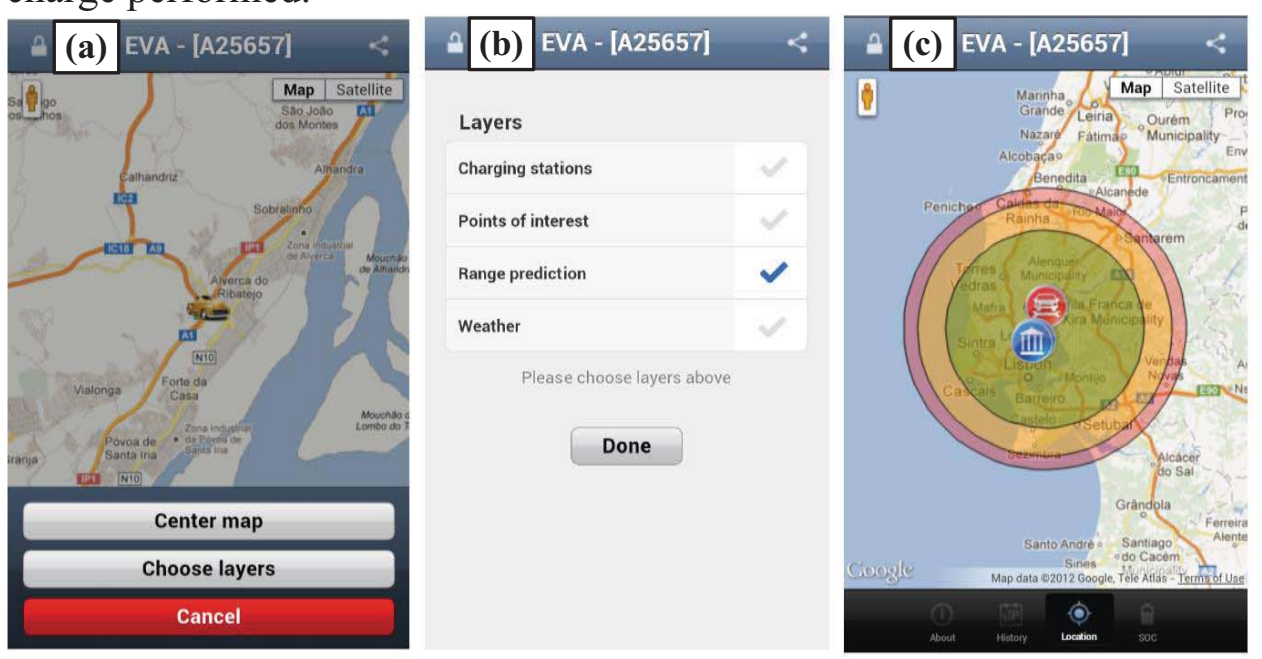

Figure 7: Range prediction interaction process: (a) EV position; (b) Available functions; (c) Range representation.

\section{External System Interface}

This functionality is related with the data integration from different information sources, such as:

1. Energy market functions, like electricity prices, broker for energy transactions, and EV aggregation platform for electricity market participation [15]. These energy transactions could be part of driver profile and can be used for automatic charging strategies taking into account past behaviour or even to charge when the prices are lower.

2. Public transportation data [16]. The access of this information is important to combine private with public transportation in the inner parts of the cities. Due to limited range and long EV battery charging time, the drivers that live far away can overcome this limitation with the use of 
public transportation. In [16] is presented the implementation of a public transportation data integration with parking facilities in a mobile device application. This solution can be used by any driver.

3. Information related with Points of Interest (POI), preloaded on the system, such that the driver can perform a quick search for POI near the present location. This information is also used for guidance to EV battery charging stations that remain at a predefined distance [16].

These systems were already described in previous publications of the authors [12][16][17].
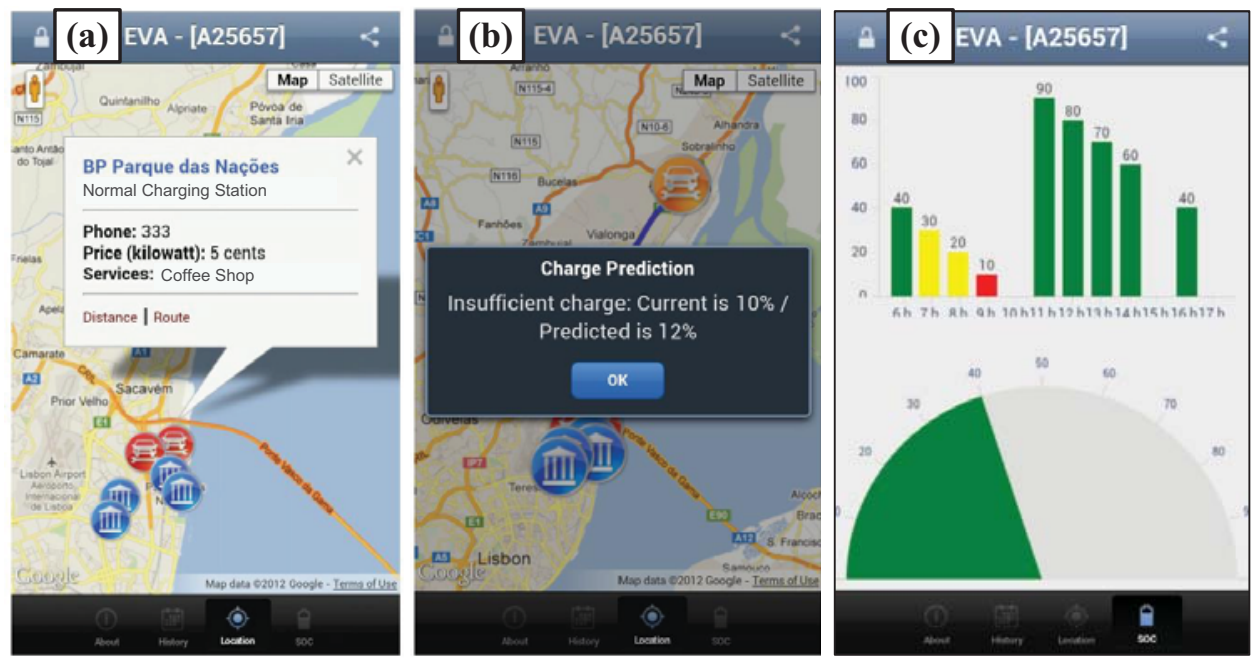

Figure 8: Screens of the EVA mobile application: (a) Guidance to Charging Stations (CS) and Points of Interest (POI); (b) Alert of insufficient charge to reach a desired destination; (c) Information about charging levels through the time and charge performed.

\section{Conclusions}

The main goal of the work presented in this paper was to create an application for monitoring of the driver behaviour and its related profile. This application is denominated as Electric Vehicle Assistant (EVA). The information stored in this profile is useful for the electric vehicle (EV) range prediction based on probabilistic approach, where is included the driver past behaviour, the EV battery state of charge (SoC), and external parameters, like road characteristics, traffic conditions and weather. Also the information from the EV driver profile can be used to establish profiles of EV driver communities (which integrate drivers with similar behaviours). Therefore, from this information, it is possible to contribute to the education of the drivers towards energy savings. The integration of EV related 
information, battery charging process, public transportation, points of interests (POI) and energy market in a mobile device, provides useful information and an integrated approach to assist EV drivers.

\section{References}

[1] João C. Ferreira, Alberto Silva and João L. Afonso. Agent based approaches for Smart charging strategy for Electric Vehicle. EVTEC 11 (1st International Electric Vehicle Tecnology Conference), 17-19 May 2011, in Yokohama, Japan.

[2] Vítor Monteiro, João C. Ferreira, , João L. Afonso, Smart Battery Charger for Electric Mobility in Smart Grids, 1st International Conference on Smart Greens, Porto-Portugal, April 2012.

[3] João C. Ferreira, Alberto Silva, Vítor Monteiro, João L. Afonso. Smart Electric Vehicle Charging System in proceedings of IEEE Intelligent Vehicles Symposium (IV 2011), June 5-9, 2011, in Baden-Baden, Germany.

[4] João C. Ferreira, A. Silva, Vítor Monteiro, João L. Afonso. "Collaborative Broker for Distributed Energy Resources," in Computational Intelligence and Decision Making, 1st ed., A.Madureira, C.Reis, V.Marques, Ed. Springer, 2012, pp.367-378.

[5] CRISP-DM Process Model. Available http://www.crispdm.org/Process/index.htm.

[6] Jose Almeida, Joao Ferreira. BUS Public Transportation System Fuel Efficiency Patterns. 2nd International Conference on Machine Learning and Computer Science (IMLCS'2013).

[7] J. Gray, A. Bosworth, A. Layman, H. Priahesh, (1995, Nov), "Data Cube: A Relational Aggregation Operator Generalizing Group-By, Cross-Tab, and Sub-Totals". Proc. 12th Int. Conf. on Data Engineering. IEEE. pp. 152-159.

[8] P Conradi. Advanced Mobile Information and Planning Support for EVs (MIPEV). (2010), Advanced Microsystems for Automotive Applications 2010, Springer.

[9] P Conradi, P Bouteiller. Dynamic Cruising Range Prediction for Electric Vehicles. (2011), Advanced Microsystems for Automotive Applications 2011, Springer.

[10] V.Figueiredo, F. Rodrigues, Z. Vale, J.B. Gouveia, (2010). An Electric Energy Consumer Characterization Framework based on Data Mining Techniques; IEEE Transactions on Power Systems; Vol. 20, n.2, pp. 596-602.

[11] J.H.Zhao, Z.Y.Dong, X.Li, K.P.Wong, (2007). A framework for electricity price spike analysis with advanced data mining methods, Power Systems, IEEE Transactions on Issue Date, vol 22, Issue 1, Feb. 2007, pp: 376 - 385. 
[12] J. C. Ferreira, A. Silva and J. L. Afonso. "EV Cockpit - Mobile Personal Travel Assistance for Electric Vehicles". Ad. Microsystems for Automotive Appli-cations 2011. Smart Systems for Electric, Safe and Networked Mobility. Series:VDI-Buch. G. Meyer, J.Valldorf(Eds.) 1st Ed, 2011, X, 344 p. ISBN 978-3-642-21380-9.

[13] A.K. Jain; M.N. Murty; P.J. Flynn, "Data Clustering: A Review" ACM Computing Surveys, Vol. 31, No. 3, September 1999.

[14] João C. Ferreira, Vítor Monteiro, João L. Afonso, "Dynamic Range Prediction for an Electric Vehicle," 27th Electric Vehicle Symposium (EVS27), Barcelona - Spain, Nov. 2013.

[15] João C. Ferreira, João L. Afonso. A Conceptual V2G Aggregation Platform in proceedings of the EVS-25 -The 25th World Battery, Hybrid and Fuel Cell Electric Vehicle Symposium \& Exhibition, 5-9 Nov. 2010, Shenzhen, China.

[16] João C. Ferreira, P. Filipe, Alberto Silva. Multi-Modal Transportation Advisor System in proceedings of the First IEEE FISTS Forum on June 29 July 1, 2011 in Vienna Austria.

[17] João C. Ferreira and João L. Afonso. Mobi_System: A Personal Travel Assistance for Electrical Vehicles in Smart Cities in proceedings of the 20th IEEE International Symposium on Industrial Electronics (ISIES 2011), 27-30 June 2011, Gdansk University of Technology, Poland. 